Original Article

\title{
Efficacy and Role of Intrathecal Fluorescein for Detection of Defect Site for Treatment of Cerebrospinal Fluid Rhinorrhea
}

\author{
Khalid Mahmood, Zafdam Shabbir, Muhammad Ishfaq, Muhammad Irfan \\ Department of Neurosurgery, Punjab Institute of Neurosciences (PINS), Lahore - Pakistan
}

\begin{abstract}
:
Background/Objectives: Cerebrospinal fluid (CSF) rhinorrhea is a rare but potentially devastating condition that can lead to significant mortality and morbidity. $90 \%$ of post-traumatic rhinorrheas stop conservatively, $10 \%$ of post-traumatic, spontaneous, and post-operative rhinorrhea need surgical repair. Fluorescein is helpful in the detection of CSF rhinorrhea peroperatively.
\end{abstract}

Material and Methods: A descriptive case series was conducted at the Department of Neurosurgery, PINS Lahore in a single unit by a single surgical team. 62 patients were enrolled who met the inclusion and exclusion criteria. The lumbar drain was passed at L4 - L5 level and $0.1 \mathrm{ml}$ of $25 \%$ sodium Fluorescein was diluted in $10 \mathrm{ml}$ of CSF. The endoscope passed through the nostril and the leakage point was detected by yellowish-green fluid coming out of the rent under endoscope. After surgery, the patients were followed up after two weeks to observe the efficacy. Post-stratification was done through a chi-square test.

Results: The mean age of the patients was 42.85 years. The most common leakage was found from cribriform plate followed by lateral lamella $\&$ sphenoid sinus. Gender difference, leakage site, and age of the patients did not have significant effects on the results of using fluorescein dye for detecting the rent. The benefit of using the dye was $79 \%$.

Conclusion: This study indicated that intrathecal fluorescein is a highly effective and beneficial approach for pinpoint localization of CSF defect, particularly very minute defects, and plays an important role in the successful treatment of CSF rhinorrhea.

Keywords: Cerebrospinal fluid (CSF) rhinorrhea, Fluorescein, Cribriform Plate, Lateral Lamella \& Sphenoid Sinus.

Corresponding Author: Muhammad Ishfaq

Department of Neurosurgery, Punjab Institute of

Neurosciences (PINS), Lahore Pakistan

Email: ishfaq141_dr@yahoo.com

Date of Submission: 01-11-2021

Date of Revision: 20-12-2021

Date of Acceptance: 22-12-2021

Date of Online Publishing: 31-12-2021

Date of Print: 31-12-2021
DOI: $10.36552 / p j n s . v 25 i 4.618$

\section{INTRODUCTION}

Cerebrospinal fluid (CSF) rhinorrhea is a rare but potentially devastating condition that can lead to significant morbidity and mortality for the patient. 
Endoscopic repair achieves more than a $90 \%$ success rate; the main constraint is identifying tiny defects intraoperatively. This issue is solved by using intrathecal fluorescein. The nasal cavity was filled with blood as a result of dissection, and a minor CSF leak was overlooked but recognized by the green-yellowish glow beneath the endoscope. Cerebrospinal fluid rhinorrhea is defined as the leaking of CSF from the subarachnoid space into the paranasal sinuses and, as a result, into the nasal cavity. It is divided mainly into traumatic and non-traumatic groups ${ }^{1-}$ 2 The traumatic group is further subdivided into two subgroups: post-procedure (iatrogenic) and post-traumatic. Leaks associated with intracranial mass lesions, hydrocephalus, empty sella syndrome, congenital abnormalities of the skull base, osteomyelitis, osteonecrosis (and other types of bone erosion), and localized cerebral atrophy are all subcategories of the nontraumatic category. CSF is as transparent as water on inspection, does not produce excoriation within or outside the nose, and individuals with CSF rhinorrhea describe the taste as salty. B2transferrin, glucose level, ring sign, and reservoir sign, all three confirm the existence of CSF. CT Functional Endoscopic Sinus Surgery Protocol (CT FESS and Magnetic Resonance Imaging Rhinorrhea Protocol) can locate the site of leakage radiographically. Following trauma, surveillance is justified since most patients resolve spontaneously. Surgical intervention is essential in traumatic situations that have not responded to medical treatment for two weeks, as well as in cases of spontaneous CSF Rhinorrhea and postoperative cases. $^{3}$

Surgery can be performed either openly or endoscopically. The open procedure is linked with significant morbidity and mortality, whereas the minimally invasive endoscopic technique has a high success rate and low morbidity and mortality. ${ }^{4-9}$ According to the literature, the overall success rate of endonasal endoscopic correction of spontaneous and traumatic CSF rhinorrhea with fluorescein dye is $80 \%{ }^{6}$ The defect in dura matter is particularly difficult to identify, especially in minor abnormalities and in the presence of blood, mucosal secretions, and clear translucent CSF. Intrathecal dyes such as indigo carmine, fluorescein, and methylene blue are used to identify leakage spots. ${ }^{5}$

Because of the low complication rate of intrathecal Sodium fluorescein, it is most usually utilized to identify and localize the dural defect. Fluorescein dye is injected into the intrathecal space via Lumber drain in this procedure, and the defect is located by watching the leaking of colored CSF via the defect. Generalized seizures, lower extremity weakness, numbness, opisthotonos, and cranial nerve deficiency have all been reported as consequences of intrathecal fluorescein administration. There is currently no local evidence on the effectiveness of intrathecal fluorescein. This research will supplement national statistics and enable for comparison with foreign literature. $^{7}$

Immediate traumatic leaks are caused by a bone defect or fracture combined with a dural rupture. A previously intact dural layer that has progressively herniated through a bone hole, eventually ripping and causing the CSF to escape is one probable cause of a delayed traumatic leak. Another view holds that the rip and bone defects are there from the start of the injury, but the leak occurs only when the masking hematoma dissolves. $^{8} \quad$ Spontaneous CSF rhinorrhea commonly appears in adulthood, coinciding with a maturational increase in CSF pressure. Because of a variety of reasons, including typical arterial and respiratory changes, the dura of the anterior cranial base is vulnerable to large variations in CSF pressure. Valsalva-like motions during nasal blowing or straining are examples of other pressures. This can result in dural rips in bone floor anomalies. 
MATERIAL AND METHODS

\section{Study Design and Setting}

A descriptive case series was conducted at the Department of Neurosurgery, Punjab Institute of Neurosciences (PINS), Lahore from 01-07-2014 to 30-12-2020.

\section{Sample Size and Sampling Technique}

The sample size of 62 cases was calculated with a $95 \%$ confidence level, $10 \%$ margin of error, and taking an expected percentage of the efficacy of endoscopic endonasal repair was $80 \%$ in spontaneous CSF Rhinorrhea. A non-probability, consecutive sampling was considered.

\section{Inclusion Criteria}

Patients of age 20 - 60 years of either gender present with Spontaneous CSF rhinorrhea. Those patients were included who developed postoperative CSF leaks or intermittent CSF leaks. Post-traumatic defects involving the midline anterior skull base (lateral lamella, cribriform plate, and fovea ethmoidalis) were also included.

\section{Exclusion Criteria}

Patients with traumatic CSF rhinorrhea in which defects are far lateral from the midline or presenting for redo surgeries were excluded, and with multiple skull base defects. Patients with rhinitis were also excluded.

\section{Clinical and Surgical Management}

62 patients fulfilling the selection criteria were enrolled in the study from the Ward of Department of Neurosurgery, PINS, and Lahore. Informed consent was obtained from the attendants of each patient. Only patients who had an endoscopic repair and received fluorescein through lumbar drain were included in the research. Radiological investigations were performed including CT scan and MRI (FESS protocol) as per our protocol preoperatively. See Figures 1 (a \& b).

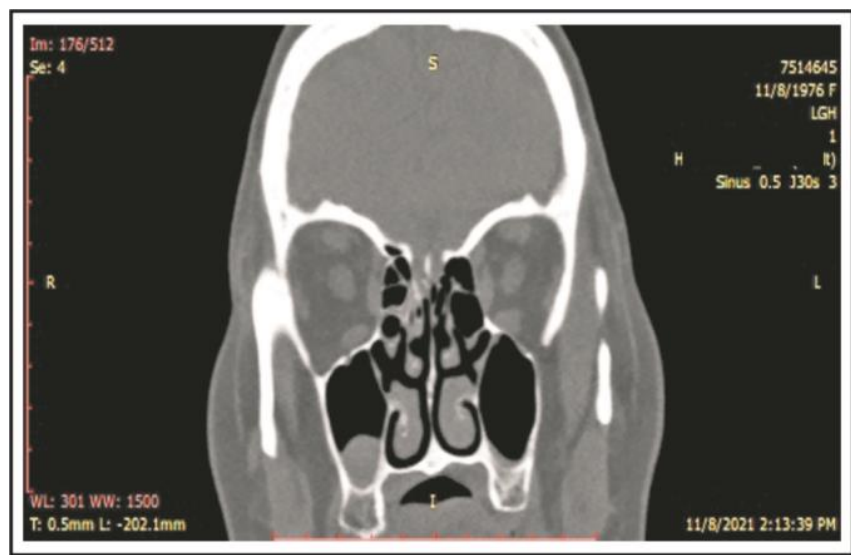

(a) Coronal View.

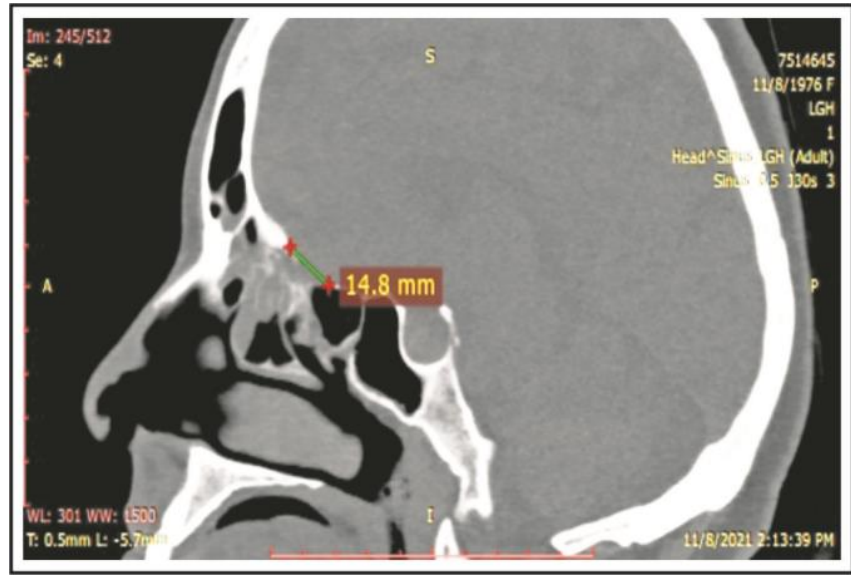

(b) Sagittal View.

Figure 1 (a \& b): A CT FESS protocol (Coronal \& Sagittal Views) and MRI CSF rhinorrhea protocol of a patient with a spontaneous CSF leak reveals a defect in the right cribriform plate. (Images used with the patient's permission).

Demographic details of the patients were also noted. Then patients were shifted to the operation theatre. All surgeries were done under general anesthesia by a single surgical team. Figure 2 shows the CSF flow down the rent. Then endoscope was passed through the nostril and leakage point was detected by yellowish-green fluid flowing out of the rent (see Figure 3). Fluorescein was identified as greenish yellowish color oozing from the defect under the endoscope. 


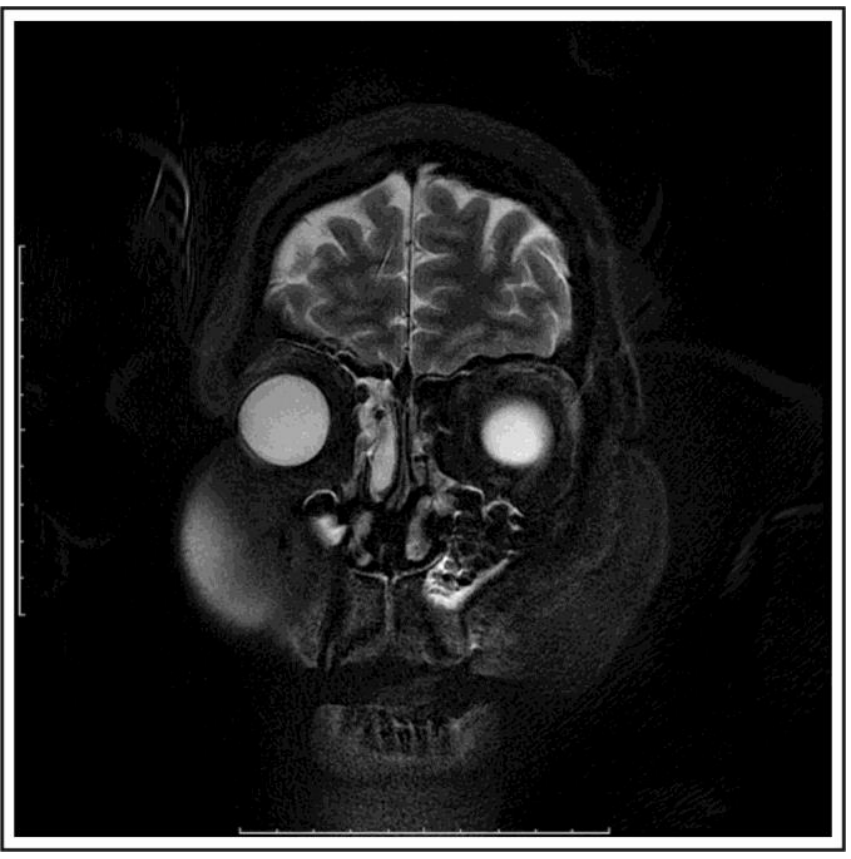

Figure 2: CSF rhinorrhea as shown by the Hyperintense leakage on MRI (brain).

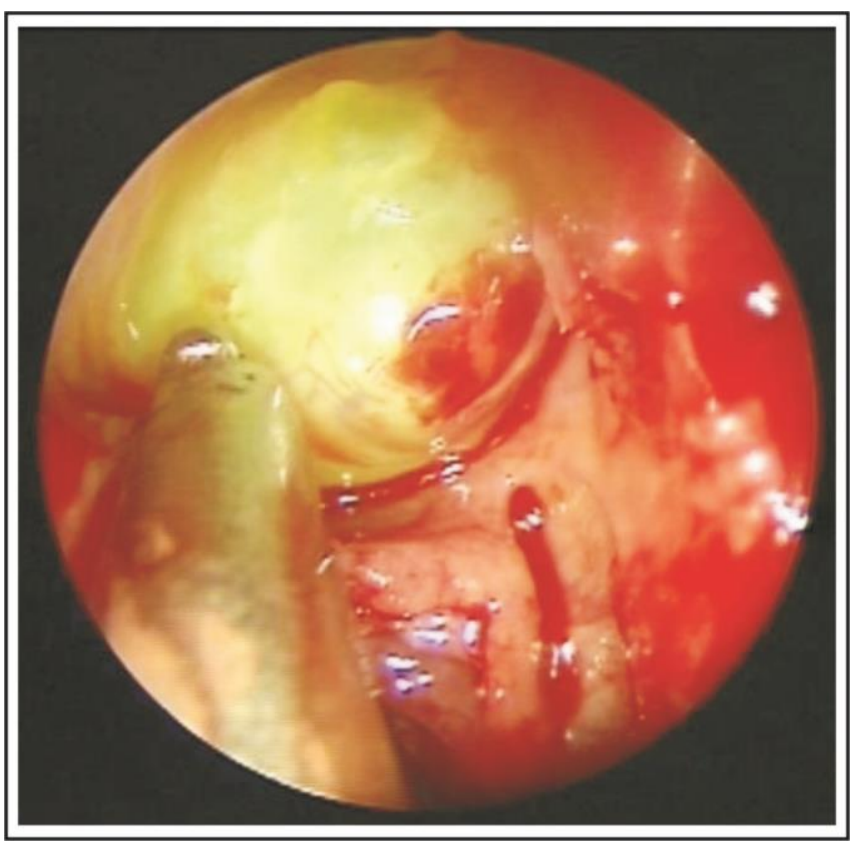

Figure 3: After intrathecal fluorescein is administered, greenish yellowish color was seen.

The rent was repaired in standard fashion by placing fascia lata graft, fat graft, and Hadad Flap in all cases and augments with Fibrin Glue and postoperatively lumber drain placed for five days.
After surgery patients were shifted to the ward and were discharged from there. Then patients were called for followed-up in the Outdoor patient department after two weeks to note the efficacy.

\section{Procedure for Intrathecal Fluorescein}

Intrathecal fluorescein has been used to identify the pinpoint the location of CSF rhinorrhea. A Lumber drain was passed at L4 - L5 level and 10 $\mathrm{ml} \mathrm{CSF}$ collected and $0.1 \mathrm{ml}$ of $25 \%$ Sodium fluorescein was diluted in $10 \mathrm{ml}$ of CSF and was re-injected into the spinal subarachnoid space in a sterile manner as a standard protocol. The fluoresce in mixed CSF before reinstallation into the subarachnoid space for 10 minutes. Then we place the patient in the Trendelenburg posture. The use of this dilution and the gradual injection method assisted to reduce central potential problems (e.g., seizures) associated with intrathecal fluorescein.

\section{Data Analysis}

The data was entered and analyzed through SPSS version 25. Mean values and standard deviations were calculated for continuous variables like age, size of rent. The percentages and frequency were calculated in the case of categorical variables like gender, and successful repair with the use of fluorescein dye. Peroperative fluorescein viability checked. Data was stratified for age, gender, and site, and size of CSF leakage to deal with effect modifiers. Post-stratification was done through chi-square test, taking $\mathrm{p}$-value $<0.05$ as significant.

\section{RESULTS}

\section{Age Distribution}

In this present study total of 62 patients with CSF rhinorrhea were enrolled. The mean age of the patients was $42.85 \pm 10.54$ years with minimum and maximum ages of $20 \& 60$ years, respectively. 


\section{Gender Distribution}

In this study, there were $23(37.10 \%)$ patients who were male, whereas 39 (62.90\%) patients were females. The male to female ratio of the patients was 0.58:1.

\section{Frequency Distribution of Leakage Site}

According to this study, leakage from lateral lamella was found in $11(17.7 \%)$ patients, leakage from the cribriform plate was noted in 46 (74.19\%) patients, and leakage from sphenoid sinus was noted in $5(8.06 \%)$ patients (see Table 1). This study's results showed that the efficacy of intrathecal fluorescein for the treatment of CSF rhinorrhea was achieved in 49(79.03\%) patients and in 13 (20\%) cases it was not observed peroperatively (Figure 4).

Table 1: Frequency distribution of leakage site.

\begin{tabular}{lcc} 
Site of Leakage & Frequency & Percent \\
Lateral lamella & 11 & 17.7 \\
Cribriform plate & 46 & 74.19 \\
Sphenoid sinus & 5 & 8.06 \\
Total & $\mathbf{6 2}$ & $\mathbf{1 0 0 . 0}$ \\
\hline
\end{tabular}

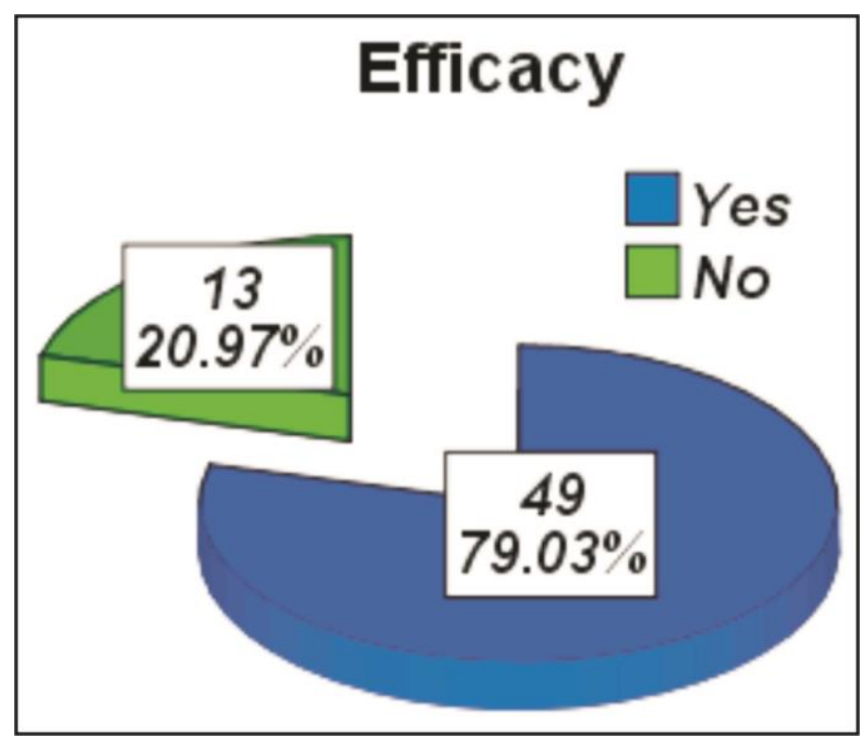

Figure 4: Frequency distribution of efficacy of intrathecal fluorescein.

\section{Comparison of Efficacy of Intrathecal Fluorescein with Age}

In our study, in patients with age $\leq 40$ years, the efficacy of intrathecal fluorescein was achieved in $21(87.5 \%)$ patients whereas, in patients with age greater than 40 years, the efficacy of intrathecal fluorescein was achieved in 28 (73.7\%) patients. Statistically, this difference was not significant ( $p$-value $=0.193)$. See Table 2.

\section{Comparison of Efficacy of Intrathecal Fluorescein with Gender}

The study results showed that among males, the efficacy of intrathecal fluorescein was achieved in 19 (82.6\%) patients whereas, among females, the efficacy of intrathecal fluorescein was achieved in $30(76.9 \%)$ patients. Statistically, this difference was not significant i.e. $p$-value $=0.595$ See Table 3.

\section{Comparison of Efficacy of Intrathecal Fluorescein with Leakage Site}

According to this study, among patients with lateral lamella defect, the efficacy of intrathecal fluorescein was achieved in 10 (91\%) patients. For the patients with cribriform leakage site, the efficacy was achieved in 36 (78.2\%) patients. Similarly, the patients with leakage sites of sphenoid sinus, the efficacy were achieved in 3 (60\%) patients. Statistically, this difference was not significant i.e. p-value $=0.359$ See Table 4 .

Gender difference, leakage site, and age of the patients did not have a significant effect on the results of using fluorescein dye for detecting the rent. The benefit of using the dye was around $79 \%$.

\section{DISCUSSION}

The goal of this descriptive case series research was to investigate the effectiveness of intrathecal fluorescein for the treatment of CSF rhinorrhea. 
Table 2: Comparison of Efficacy of Intrathecal Fluorescein with Age (Years).

\begin{tabular}{|cccccc} 
& & \multicolumn{2}{c}{ Efficacy } & Total & p-value and Chi-Square \\
Age (Years) & $\leq 40$ & $21(87.5 \%)$ & $3(12.5 \%)$ & $24(100.0 \%)$ & p: 0.193 \\
Total & $>40$ & $28(73.7 \%)$ & $10(26.3 \%)$ & $38(100.0 \%)$ & (insignificant) \\
\hline
\end{tabular}

Table 3: Comparison of Efficacy of Intrathecal Fluorescein with Gender.

\begin{tabular}{|c|c|c|c|c|c|}
\hline & & \multicolumn{2}{|c|}{ Efficacy } & \multirow{2}{*}{ Total } & \multirow{2}{*}{$\begin{array}{l}\text { p-value and } \\
\text { Chi-Square }\end{array}$} \\
\hline & & Yes & No & & \\
\hline \multirow{2}{*}{ Gender } & Male & 19 (82.6\%) & 4 (17.4\%) & 23 (100.0\%) & p: 0.595 \\
\hline & Female & 30 (76.9\%) & 9 (23.1\%) & 39 (100.0\%) & (insignificant) \\
\hline \multicolumn{2}{|c|}{ Total } & 49 (79.0\%) & $13(21.0 \%)$ & 62 (100.0\%) & $x^{2}=0.28$ \\
\hline
\end{tabular}

Table 4: Comparison of Efficacy of Intrathecal Fluorescein with Leakage Site.

\begin{tabular}{|c|c|c|c|c|}
\hline \multirow{2}{*}{ Leakage Site } & \multicolumn{2}{|c|}{ Efficacy } & \multirow{2}{*}{ Total } & \multirow{2}{*}{ p-value and Chi-Square } \\
\hline & Yes & No & & \\
\hline Lateral lamella & 10 (90.9\%) & 1 (9.09\%) & 11 (100.0\%) & \\
\hline Cribriform plate & 36 (78.2\%) & 10 (21.7\%) & 46 (100.0\%) & cignifir \\
\hline Sphenoid sinus & $3(60 \%)$ & $2(40 \%)$ & 5 (100.0\%) & nsignirican \\
\hline Total & 49 (79.0\%) & 13 (21.0\%) & 62 (100.0\%) & $x^{2}=$ \\
\hline
\end{tabular}

The presence of CSF within the nasal cavity as a result of lack of barrier between the subarachnoid space and the extracranial space is referred to as CSF Rhinorrhea. CSF rhinorrhea occurs when there is a fistula between the dura and the skull base, resulting in CSF discharge from the nose. Many scientific publications have been generated that convincingly demonstrate the therapeutic utility of intrathecal fluorescein..$^{8-}$ 10

We observed that the gender difference, location of the leak, and age of the patients did not affect the outcomes of applying fluorescein dye to identify the rent. The advantage of employing the dye in identifying the defect was around 79 percent. In our first two or three cases, we discovered that finding the specific leaking location, even using an endoscope, was challenging, resulting in excessive tissue removal, especially in minor defects. Later, we began utilizing fluorescein in a low dosage of $25 \mathrm{mg}$ per
$10 \mathrm{ml}$ through a lumbar drain. It is especially useful for tiny faults in pinpoint localization. The fluorescence of yellowish greenish fluorescein beneath the endoscope easily identifies defects. The maximum advised dose for fluorescein usage in the literature was $50 \mathrm{mg}$; we used a $25 \mathrm{mg}$ dose in all of our cases, and no complications such as fits or other side effects were seen. Fluorescein was not visible under endoscope in our 13 cases, despite proper lumber drain placement in subarachnoid space and proper Trendelenburg position after fluorescein introduction in subarachnoid space, in our opinion after careful literature review. This could be due to arachnoid scarring post meningitis, lumbar stenosis, or insufficient time after intrathecal injection for CSF circulation, but we were unable to comment properly why fluorescein was not visible under endoscope. One perspective is that in such circumstances, a large dosage of fluorescein should be used, however 
owing to the major complications indicated in the literature, we were extremely cautious in employing small doses in our entire series.

The effectiveness of intrathecal fluorescein for the treatment of CSF rhinorrhea was attained in 49 (79.03 percent) of the patients in our research. Javadi et al $^{11}$ researched the use of low-dose intravenous fluorescein in the endoscopic correction of CSF rhinorrhea. Intravenous fluorescein injection revealed CSF leakage in 18 of 20 of the participants in their research. There were no difficulties throughout the procedure. They proposed that administering a modest dose of intrathecal fluorescein is a safe and sensitive approach for locating CSF leakage.

Seth et $\mathrm{al}^{12}$ investigated the use of intrathecal fluorescein in CSF leak correction. They found that using intrathecal fluorescein aids in the correct location of CSF leaks and may help the surgeon ensure a watertight closure. Because of the low complication rate of intrathecal Sodium fluorescein, it is most usually utilized to identify and localize the dural defect. Sodium fluorescein dye was introduced into the intrathecal space via Lumber drain in this procedure, and the defect was located by watching the leaking of colored CSF through the defect. This method has a 1.9 percent recurrence rate.

In their investigation, Felisati et $\mathrm{al}_{1}{ }^{13}$ concluded that lumbar intrathecal fluorescein administration is a safe method if the following conditions are met: maximum dosage of $50 \mathrm{mg}$ of fluorescein, further dilution of the colorant in CSF, and gradual delivery. Except for a few isolated cases, the literature demonstrates the test's use, both in the diagnostic and intra-operative phases, as well as for preventative reasons, when surgical operations on the skull base are done with the risk of dural injury.

Placantonakis et $\mathrm{al}_{1}{ }^{14}$ discovered that lowdose intrathecal fluorescein injection following premedication with steroid and antihistamine drugs is often safe. The majority of symptoms is generic and temporary and is most likely caused by the operation or lumbar drainage. There is not a lot of local data on this subject. According to this study, leakage from the lateral lamella was identified in 11 (17.7\%) patients, leakage from the cribriform plate was detected in 36 patients, and leakage from the sphenoid sinus was found in 15 (24.2\%) patients.

When it comes to the location of leakage, one research by Virk et al. ${ }^{15}$ found that fifty percent of spontaneous leaks came from the cribriform plate, 22 percent from the sphenoid, 14 percent from the ethmoid, and 14 percent from the frontal sinus. In the traumatic CSF leak group, 33.3 percent were from the cribriform plate, 33.3 percent were from the sphenoid, 22.2 percent were from the ethmoid, and 11.1 percent were from the frontal. Lumbar drains and antibiotics are utilized as adjuvant therapy to endoscopic repair, although their advantages are unclear; intrathecal fluorescein can be used to help in CSF leak detection but should be reserved for more difficult situations.

According to the research, endoscopic correction of CSF rhinorrhea is safe and effective, with a very low complication risk. It has practically completely replaced traditional open techniques. ${ }^{16}$ According to the current study, intrathecal fluorescein was effective in 19 (82.6 percent) of male patients and 30 (76.9 percent) of female patients. There are certain limitations to this study, such as the fact that endoscopic repair is not adequately common in our nation, therefore there is very little data on its utilization, and it is only available in centers that do routine endoscopic surgery.

\section{CONCLUSION}

This study indicated that intrathecal fluorescein is a highly effective and beneficial approach for pinpoint localization of CSF abnormalities, particularly very minute defects, and plays an important role in the successful treatment of CSF rhinorrhea. The gender difference, location of the 
leak, and age of the patients did not affect the outcomes of fluorescein dye to identify the rent. The advantage of fluorescein dye preoperatively was around 79 percent.

\section{REFERENCES}

1. Aydin K, Terzibasioglu E, Sencer $S$, Sencer A, Suoglu $Y$, Karasu $A$, et al. Localization of cerebrospinal fluid leaks by gadolinium-enhanced magnetic resonance cisternography: a 5-year single-center experience. Neurosurgery, 2008; 62 (3): 584-9.

2. Deenadayal $D$, Vidyasagar $D$, Kumar $M N$, Sudhakshin P, Chandra SS, Hameed S. Spontaneous CSF rhinorrhea our experience. Indian Journal of Otolaryngology and Head \& Neck Surgery, 2013; 65 (2): 271-5.

3. Mark S. Greenberg. Hydrocephalus and cerebrospinal Fluid. In: Mark S. Greenberg eHontNYT.

4. Banu MA, Kim JH, Shin BJ, Woodworth GF, Anand $\mathrm{VK}$, Schwartz TH. Low-dose intrathecal fluorescein and etiology-based graft choice in endoscopic endonasal closure of CSF leaks. Clinical Neurology and Neurosurgery, 2014; 116: 28-34.

5. Mark S. Greenberg. Procedures IOIMSG, ed. Handbook of neurosurgery. $8^{\text {th }}$ ed: New York: Thieme, 2016: 1426.

6. Mishra SK, Mathew GA, Paul RR, Asif SK, John M, Varghese AM, Kurien M. Endoscopic repair of CSF rhinorrhea: an institutional experience. Iranian Journal of Otorhinolaryngology, 2016; 28 (84): 39.

7. Kong X, Guan J, Yang Y, Li Y, Ma W, Wang R. A meta-analysis: Do prophylactic antiepileptic drugs in patients with brain tumors decrease the incidence of seizures? Clinical Neurology and Neurosurgery, 2015; 134: 98-103.
8. Abuabara A. Cerebrospinal fluid rhinorrhoea: diagnosis and management. Medicina Oral, Patología Oral y Cirugía Bucal (Internet), 2007; 12 (5): 397-400.

9. Fridley J, Foroozan R, Sherman V, Brandt ML, Yoshor D. Bariatric surgery for the treatment of idiopathic intracranial hypertension: a review. Journal of Neurosurgery, 2011; 114 (1): 34-9.

10. Jones ME, Reino T, Gnoy A, Guillory S, Wackym P, Lawson W. Identification of intranasal cerebrospinal fluid leaks by topical application with fluorescein dye. American Journal of Rhinology, 2000; 14 (2): 93-6.

11. Javadi SAH, Samimi H, Naderi F, Shirani M. The use of low-dose intrathecal fluorescein in endoscopic repair of cerebrospinal fluid rhinorrhea. Archives of Iranian Medicine, 2013; 16 (5): 264.

12. Seth R, Rajasekaran K, Benninger MS, Batra PS. The utility of intrathecal fluorescein in cerebrospinal fluid leak repair. Otolaryngology-Head and Neck Surgery, 2010; 143 (5): 626-32.

13. Felisati G, Bianchi A, Lozza P, Portaleone S. Italian multicentre study on intrathecal fluorescein for craniosinusal fistulae. Acta Otorhinolaryngologica Italica. 2008; 28 (4): 159.

14. Placantonakis DG, Tabaee A, Anand VK, Hiltzik D, Schwartz TH. Safety of low-dose intrathecal fluorescein in endoscopic cranial base surgery. Operative Neurosurgery, 2007; 61 (suppl_3): ONS161.

15. Virk JS, Elmiyeh B, Saleh HA. Endoscopic management of cerebrospinal fluid rhinorrhea: the charing cross experience. Journal of neurological surgery Part B, Skull Base, 2013; 74 (2): 61.

16. Sharma S, Kumar G, Bal J, Eweiss A. Endoscopic repair of cerebrospinal fluid rhinorrhoea. European annals of otorhinolaryngology, head and neck diseases, 2016; 133 (3): 187-90.

\section{Additional Information}

Disclosures: Authors report no conflict of interest.

Ethical Review Board Approval: The study was conformed to the ethical review board requirements.

Human Subjects: Consent was obtained by all patients/participants in this study.

Conflicts of Interest:

In compliance with the ICMJE uniform disclosure form, all authors declare the following: 
Financial Relationships: All authors have declared that they have no financial relationships at present or within the previous three years with any organizations that might have an interest in the submitted work.

Other Relationships: All authors have declared that there are no other relationships or activities that could appear to have influenced the submitted work.

\section{AUTHORS CONTRIBUTIONS}

\begin{tabular}{|l|l|l|}
\hline Sr.\# & Author's Full Name & Intellectual Contribution to Paper in Terms of: \\
\hline 1. & Khalid Mahmood & 1. $\quad$ Study design and methodology. \\
\hline 2. & Muhammad Ishfaq & 2. Paper writing, referencing and data calculations. \\
\hline 3. & Zafdam Shabbir & 3. Data collection and calculations. \\
\hline 4. & Muhammad Ishfaq & 4. Analysis of data and interpretation of results etc. \\
\hline 5. & Muhammad Irfan & 5. Literature review and manuscript writing. \\
\hline 6. & Khalid Mahmood & 6. Analysis of data and quality insurer. \\
\hline
\end{tabular}

